\title{
Diffraction phases in atom interferometers
}

\author{
M. Büchner, R. Delhuille, A. Miffre, C. Robilliard, J. Vigué (a) \\ and C. Champenois (b) \\ (a) Laboratoire Collisions Agrégats Réactivité -IRSAMC \\ Université Paul Sabatier and CNRS UMR 5589, 118, \\ Route de Narbonne 31062 Toulouse Cedex, France \\ (b) PIIM, Université de Provence and CNRS UMR 6633, \\ Centre de saint Jérôme case C21, \\ 13397 Marseille cedex 20, France. \\ e-mail: jacques.vigue@irsamc.ups-tlse.fr
}

\begin{abstract}
Diffraction of atoms by laser is a very important tool for matter wave optics. Although this process is well understood, the phase shifts induced by this diffraction process are not well known. In this paper, we make analytic calculations of these phase shifts in some simple cases and we use these results to model the contrast interferometer recently built by the group of D. Pritchard at MIT. We thus show that the values of the diffraction phases are large and that they probably contribute to the phase noise observed in this experiment.
\end{abstract}

PACS numbers: $03.75 . \mathrm{Dg}, 39.20 .+\mathrm{q}, 32.80 . \mathrm{Pj}, 42.50 . \mathrm{Vk}$

\section{INTRODUCTION}

In atom interferometry, laser diffraction is a very powerful and versatile tool (for overviews, see references [1. 2]). The diffraction of matter waves by a standing light wave was proposed by P. Kapitza and P.A.M. Dirac [3] in the case of electrons and generalized to atoms by S. Altshuler et al. [四. Atom diffraction by light has been studied theoretically [5, 6] and experimentally [7, 8] and these early works have been followed by many studies too numerous to be quoted here. The phases of the diffraction amplitudes are rarely discussed in detail, with a few exceptions like the works of S. Chu and coworkers [9] and of K. Burnett and coworkers [10], in both cases for Raman adiabatic transfer, and the work of C. Bordé and coworkers [1], 12], which analyzes the general diffraction process in the rotating wave approximation. Unfortunately, this approximation cannot be used for elastic diffraction studied here.

In an interferometer, the diffraction phases modify the interference signals but this effect is difficult to detect, as it requires accurate phase measurements and it cancels in symmetric interferometers, like the Mach-Zehnder interferometer. The goal of this paper is to present an analytic calculation of diffraction phases in a simple case (elastic diffraction by a laser standing wave) and to show the importance of these diffraction phases in an existing experiment. We consider here diffraction in the RamanNath regime and second order Bragg diffraction in the weak field regime and we apply these results to the contrast interferometer built by the group of D. Pritchard [13. The calculated diffraction phases are large in this interferometer and as these phases depend rapidly on the laser power density used for the diffraction process, our calculation may explain the observed phase noise as resulting from fluctuations of this power density.

\section{THE PROBLEM}

We consider diffraction of slow ground state atoms by a near-resonant laser standing wave of frequency $\omega_{L}$. For a sufficiently large laser detuning $\delta=\omega_{L}-\omega_{0}$, where $\omega_{0}$ is the resonance transition frequency, the probability of real excitation is negligible and the diffraction process is coherent. In the dressed-atom picture [14, the laser standing wave creates a light shift potential $V(x, t)$ :

$$
\begin{aligned}
V(x, t) & =V_{0}(t) \cos ^{2}\left(k_{L} x\right) \\
& =\frac{V_{0}(t)}{4}\left[2+\exp \left(+2 i k_{L} x\right)+\exp \left(-2 i k_{L} x\right)\right](1)
\end{aligned}
$$

where the envelope $V_{0}(t)$ is proportional to the laser power density divided by the frequency detuning $\delta$ and $k_{L}$ is the laser wavevector. We are going to forget the $x$-independent term, which simply shifts the energy zero and therefore has no effect, as long as all atoms see the same potential. The motion along the $y$ and $z$ directions is free and will not be discussed. The natural energy unit is the atom recoil energy $\hbar \omega_{\text {rec }}=\hbar^{2} k_{L}^{2} / 2 m$ and we will measure the potential with this unit, by defining $q(t)$ [15, 16] :

$$
q(t)=V_{0}(t) /\left(4 \hbar \omega_{r e c}\right)
$$

Using a dimensionless time $\tau$ defined by $\tau=\omega_{\text {rec }}$, a dimensionless spatial coordinate, $X=k_{L} x$ and a dimensionless wavevector $\kappa=k_{x} / k_{L}$, the 1D Schrödinger equation becomes :

$$
i \frac{\partial \Psi}{\partial \tau}=-\frac{\partial^{2} \Psi}{\partial X^{2}}+q(\tau)[\exp (2 i X)+\exp (-2 i X)] \Psi
$$

For a constant value of the potential $q$, the atom eigenstates are Bloch states [15, 16, 17]. Writing the Hamiltonian matrix corresponding to equation (3) in the basis $|\kappa\rangle$ of plane waves of momentum $\hbar \kappa$ and using numerical 
diagonalization, we get the band structure $\varepsilon(\kappa, p)$, with the pseudo-momentum $\kappa$ belonging to the first Brillouin zone $(-1<\kappa \leq 1)$ and the integer $p$ labeling the bands [16]. Figure 1 presents the energy of the lowest Bloch states as a function of $\kappa$ for two values of the potential, $q=0$ and $q=1$, with two important features : when $q$ is not equal to zero, band gaps appear at each crossing of the $q=0$ folded parabola and energy shifts appear at the same time. These energy shifts are explained by perturbation theory : each free plane wave $|\kappa\rangle$ is coupled to two other states, $|\kappa \pm 2\rangle$ and the two coupling terms are equal. As the energy denominator is larger for the coupling to the upper state, all the levels are pushed upwards (except near the places where gaps open), but the lowest Bloch state is obviously pushed downwards.

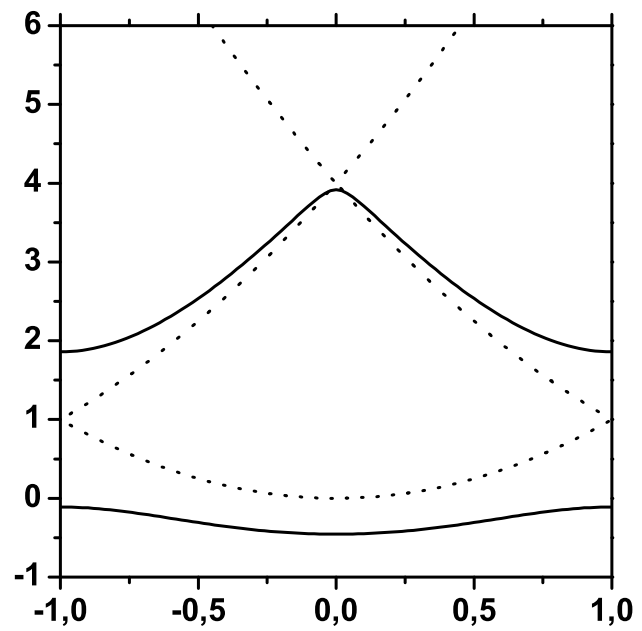

FIG. 1: Plots of the energies $\varepsilon$ of the lowest Bloch states versus the pseudomomentum $\kappa$ : solid line $q=1$; dashed line $q=0$.

\section{DIFFRACTION PHASES}

In order to simplify the calculations, we consider that the atom is initially in a state of zero momentum, $|\psi(\tau=0)\rangle=|0\rangle$. We first consider diffraction in the Raman-Nath regime. This approximation consists in neglecting the dynamics of the atom during the diffraction process produced by a pulse $q(\tau)$ of duration $\tau_{R N}$. This approximation is good if the potential $q(\tau)$ is intense, $q \gg 1$, and if the pulse is brief, $\tau_{R N} \ll 1$. The validity range of this approximation is given by [15, 16] :

$$
\tau_{R N}<1 /(4 \sqrt{q})
$$

and the diffracted wave is a classic result :

$$
\left|\psi\left(\tau_{R N}\right)\right\rangle=\sum_{p}(-i)^{|p|} J_{|p|}(\gamma)|2 p\rangle
$$

with $\gamma=2 q \tau_{R N}$. We have verified [16] that the RamanNath formula predicts accurately the diffraction probability of order 0 and 1 , for finite values of the parameter $q$, as long as condition (4) is verified, but we have not tested the phases of these diffraction amplitudes. They could be tested by using the diffraction amplitudes calculated [18] as a power series of $1 / q$.

Second order Bragg diffraction is due to the indirect coupling of the $| \pm 2\rangle$ free states, through the $|0\rangle$ state. As this coupling is a second order term in $q$, to make a consistent treatment, we must consider the 5 lowest energy states, with $\kappa=0, \pm 2, \pm 4$. The Hamiltonian matrix has the following non-vanishing elements $\langle 2 p|H| 2 p\rangle=4 p^{2}$ and $\langle 2 p|H| 2(p \pm 1)\rangle=q$. Up to second order in $q$, the energy correction of the $\mid 0>$ state is $E_{0}=-q^{2} / 2$ and the effective Hamiltonian coupling the states $|-2\rangle$ and $|+2\rangle$ is:

$$
H_{e f f}=\left[\begin{array}{cc}
4+\left(q^{2} / 6\right) & \left(q^{2} / 4\right) \\
\left(q^{2} / 4\right) & 4+\left(q^{2} / 6\right)
\end{array}\right]
$$

We have tested the quality of this expansion limited to the $q^{2}$ terms, by numerical diagonalization of the Hamiltonian matrix. The neglected terms (in $q^{4}$, etc.) are of the order of $1 \%(10 \%)$ of the $q^{2}$ terms if $q=0.3(q=1$ respectively), thus giving an idea of the validity range of this calculation.

The dynamics is adiabatic if the potential $q(\tau)$ varies slowly, but diffraction remains possible when two free states are degenerate, as the $| \pm 2\rangle$ states. The problem is equivalent to a Rabi oscillation exactly at resonance, for which an exact solution is available for any function $q(\tau)$. For a pulse extending from $\tau_{1}$ to $\tau_{2}$, the Rabi phase $\varphi_{r}$ at the end of the pulse is given by :

$$
\varphi_{r}=\int_{\tau_{1}}^{\tau_{2}}\left(q^{2} / 2\right) d \tau
$$

and if $\left|\psi\left(\tau_{1}\right)\right\rangle=| \pm 2\rangle$, the final state is :

$$
\begin{aligned}
\left|\psi\left(\tau_{2}\right)\right\rangle & =e^{\left[-i\left(4\left(\tau_{2}-\tau_{1}\right)+\left(\varphi_{r} / 3\right)\right)\right]} \\
& \times\left[\cos \left(\frac{\varphi_{r}}{2}\right)| \pm 2\rangle-i \sin \left(\frac{\varphi_{r}}{2}\right)|\mp 2\rangle\right]
\end{aligned}
$$

where the phase shift of the $\mid \pm 2>$ states due to theirmean energy shift has been expressed as a fraction of the Rabi phase. When $\left|\psi\left(\tau_{1}\right)\right\rangle=|0\rangle$, the final state is the $|0\rangle$ state with an extra phase shift, also due to its energy shift:

$$
\left|\psi\left(\tau_{2}\right)\right\rangle=e^{i \varphi_{r}}|0\rangle
$$

From now on, we consider a $\varphi_{r}=\pi$ pulse. If the wavefunction at time $\tau_{1}$ is given by :

$$
\left|\psi\left(\tau_{1}\right)\right\rangle=\sum_{p=-2,0,+2} a_{p}\left(\tau_{1}\right)|p\rangle
$$

the wavefunction at time $\tau_{2}$ is given by :

$$
\begin{aligned}
\left|\psi\left(\tau_{2}\right)\right\rangle & =e^{i \pi} a_{0}\left(\tau_{1}\right)|0\rangle+e^{\left[-4 i\left(\tau_{2}-\tau_{1}\right)-(5 i \pi / 6)\right]} \\
& \times\left[a_{-2}\left(\tau_{1}\right)|+2\rangle+a_{+2}\left(\tau_{1}\right)|-2\rangle\right]
\end{aligned}
$$


The phase factor $\exp \left[-4 i\left(\tau_{2}-\tau_{1}\right)\right]$ is due to the free propagation of the $\mid \pm 2>$ states and is not linked to the diffraction process. The interesting results are the diffraction phases equal to $(+\pi)$ for the $|0\rangle$ state and $(-5 \pi / 6)$ for the $| \pm 2\rangle$ states. The opposite signs of the diffraction phases are a consequence of the opposite signs of the energy shifts of these levels. In the resulting phase difference, the level shift contribution, equal to $4 \pi / 3$, is proportional to the Rabi phase $\varphi_{r}$, taken equal to $\pi$. In an experiment, this phase difference may differ from this calculated value, as a result of an imperfect $\pi$ pulse or of other effects neglected here (e. g. : $\kappa \neq 0$ ).

\section{SIMPLE MODEL OF THE CONTRAST INTERFEROMETER OF S. GUPTA ET AL}

We now calculate the output signal of the contrast interferometer developed by S. Gupta et al. [13]. This interferometer uses second order Bragg diffraction and Raman-Nath diffraction and the atomic paths are represented in figure 2. The initial state is a Bose Einstein condensate, approximated here by a $|\kappa=0\rangle$ state. A first intense and brief pulse from $\tau=0$ till $\tau_{R N}$ is used to diffract this initial state in three coherent states, $|0\rangle$, $| \pm 2\rangle$. Within the Raman-Nath approximation, the wavefunction for $\tau_{R N}$ is given by :

$$
\left|\psi\left(\tau_{R N}\right)\right\rangle=J_{0}|0\rangle-i J_{1}[|+2\rangle+|-2\rangle]
$$

the argument $\gamma$ of Bessel functions being omitted for compactness. The best contrast [13] would be obtained with diffraction probabilities equal to $50 \%$ for the $|0\rangle$ state and $25 \%$ for each of the $| \pm 2\rangle$ states. It is impossible to fulfill perfectly these two conditions simultaneously as the first one implies $\gamma=1.13$ whereas the second one implies $\gamma=1.21$. We can nevertheless suppose that $\gamma \approx 1.17$. Although $J_{2}(1.17) \approx 0.15$, we will neglect here the second order diffraction amplitudes, as done in reference $\left[13\right.$. We assume that $\tau_{R N}$ is negligible so that free propagation starts at $\tau=0$ and lasts till the Bragg diffraction pulse which extends from $\tau_{1}$ to $\tau_{2}$. Using equation (11), we get the wavefunction after this pulse :

$$
\begin{aligned}
\left|\psi\left(\tau_{2}\right)\right\rangle & =J_{0} e^{i \pi}|0\rangle \\
& +J_{1} e^{-4 i \tau_{2}} e^{-4 i \pi / 3}[|+2\rangle+|-2\rangle]
\end{aligned}
$$

Free propagation goes on till a time $\tau$ where the matter grating formed by the interference of these three states is read by the reflection of a laser beam. The atomic density as a function of $X$ and $\tau$ is deduced from the wavefunction :

$$
\begin{aligned}
|\langle X \mid \psi(\tau)\rangle|^{2} & =J_{0}^{2}+2 J_{1}^{2}(1+\cos (4 X))+4 J_{0} J_{1} \\
& \times \cos (2 X) \cos \left(4 \tau+\frac{7 \pi}{3}\right)
\end{aligned}
$$

The experimental signal $S(\tau)$ is the intensity of the light reflected by this grating. This homodyne detection signal is proportional to the square of the $\cos (2 X) \bmod -$ ulation of the atomic density, with the following timedependence:

$$
S(\tau) \propto \cos ^{2}\left(4 \tau+\frac{7 \pi}{3}\right)
$$

while the equation used by Gupta et al. is :

$$
S(\tau) \propto \sin ^{2}(4 \tau)
$$

The difference between equation (15) and (16) will be important only if one wants to make an absolute prediction of the phase, but it has no consequence in the analysis carried by S. Gupta et al. [13], because their fitted value of $\omega_{\text {rec }}$ comes from the derivative of the phase with the time interval $T$ [19]. However, our result remains interesting as it may explain a large part of the observed phase noise, $200 \mathrm{mrad}$ from shot to shot. In the $7 \pi / 3$ phase of equation (15), $4 \pi / 3$ are proportional to the Rabi phase, which is itself proportionnal to $q^{2}$ i.e. to the square of the laser power density during the Bragg pulse. Therefore, a $1 \%$ variation of the laser power density changes the diffraction phase by $84 \mathrm{mrad}$.

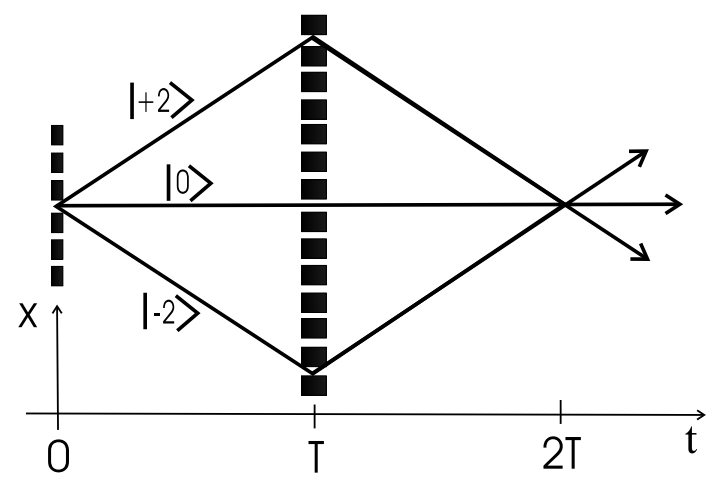

FIG. 2: In the $x, t$ plane, we have represented the atomic paths followed by the wavepackets in the interferometer of Gupta et al. [13] : Raman-Nath diffraction at time $t=0$, second order Bragg diffraction at time $t=T$, detection near time $t=2 T$.

Our calculation relies on several approximations, some of them being not very accurate in the experimental conditions of S. Gupta et al. [13:

i) the $\kappa=0$ approximation is an oversimplification but the calculation with $\kappa \neq 0$ is more complex.

ii) the first diffraction pulse used in the experiment is $1 \mu \mathrm{s}$ long, corresponding to $\tau_{R N}=0.157$. Assuming $\gamma \approx 1.17$, we get $q \approx 3.7$ and the validity condition (4) requires $\tau \leq 0.13$. Therefore, the corrections to the Raman-Nath phases are not fully negligible. We have also neglected the second order diffraction beams, which contribute to the signal.

iii) as for the perturbation expansion used to describe Bragg diffraction, the $\pi$-pulse used is Gaussian with a 
width of $7.6 \mu \mathrm{s}[13]$. Assuming that $q=q_{\max } \exp [-(t-$ $\left.T)^{2} /\left(2 \sigma_{t}^{2}\right)\right]$, with $\sigma_{t}=3.8 \mu$ s, i.e. $\sigma_{\tau} \approx 0.6$, we get the value $q_{\max } \approx 2.4$, well outside the validity range of our second order perturbation expansion. Higher order terms in $q^{n}$ with $n=4,6, .$. contribute to the phases and the sensitivity of the diffraction phase to the laser power density may even be larger than predicted above.

Obviously, to describe very accurately this experiment, a full numerical modelization is needed and feasible, as the problem reduces to a $1 D$ Schrödinger equation, if atom-atom interactions are neglected. But, as noted by Gupta et al., the mean field effect of the condensate can also modify atomic propagation and this effect has not been not considered here.

\section{CONCLUSION}

In this paper, we have made a simple and tutorial calculation of the phase shifts of atomic waves due to elastic diffraction process by a laser standing wave. We have calculated the associated phase shift for the contrast interferometer of D. Pritchard et al. [13], thus showing that it should be possible to make an experimental test of the dependence of the diffraction phase shifts with potential strength and interaction time. The present calculations are simple because of our assumptions : Raman-Nath limit or perturbative regime, vanishing initial momentum $\kappa=0$. An accurate modelization of a real experiment requires numerical integration of Schrödinger equation to describe the diffraction dynamics without any approximation.
We have considered only first and second order diffraction. Higher diffraction orders up to order 8, have been observed [20, 21, 22] with moderate laser power densities. The leading term of the coupling matrix element responsible for diffraction order $n$ behaves like $q^{n}$ 20] whereas the leading terms of the energy shifts, responsible for the diffraction phase shifts, are always in $q^{2}$. Therefore, for diffraction orders $n>2$, the control of the phase shifts will require a full knowledge of the pulse shape. For the second order of diffraction, the diffraction phase shifts and the Rabi phase are simply related, as long as second order perturbation theory is a good approximation.

We have made a systematic use of atomic Bloch states to describe atom diffraction by laser, following our previous paper [16]. The introduction of Bloch states to describe atoms in a laser standing waves is due to Letokhov and Minogin 23, 24 in 1978 and also to Castin and Dalibard 25] in 1991. Their use is rapidly expanding, in particular to treat Bose-Einstein condensates in an optical lattice, as reviewed by Rolston and Phillips [26. When coupled to reduced units as done here, the atomic Bloch states represent a very efficient tool to get a simple understanding of the diffraction process.

\section{ACKNOWLEDGEMENTS}

We thank C. Cohen-Tannoudji, J. Dalibard and C. Salomon for very fruitfull discussions, D. Pritchard for a very useful private communication and Région Midi Pyrénées for financial support.
[1] Atom interferometry edited by P. R. Berman (Academic Press 1997)

[2] Bose-Einstein condensate and atom lasers, Atom optics and interferometry, A. Aspect and J. Dalibard ed., C. R. Acad. Sc. Paris, 2, série IV, 333-693 (2001)

[3] P. L. Kapitza and P. A. M. Dirac, Proc. Cambridge Phil. Soc., 29, 297-300 (1933)

[4] S. Altshuler, L. M. Frantz and R. Braunstein, Phys. Rev. Lett. 17, 231 (1966)

[5] R. J. Cook and A. F. Bernhardt, Phys. Rev. A 18, 2533 (1978)

[6] A. F. Bernhardt and B. W. Shore, Phys. Rev. A 23, 1290 (1981)

[7] E. Arimondo, H. Lew and T. Oka, Phys. Rev. Lett., 43, 753 (1979)

[8] P.E. Moskowitz et al., Phys. Rev. Lett., 51, 370 (1983)

[9] M. Weitz, B. C. Young and S. Chu, Phys. Rev. A 50, 2438 (1994)

[10] P. D. Featonby et al., Phys. Rev. A 53, 373 (1996)

[11] Ch. J. Bordé, in ref. [1], p. 257

[12] Ch. J. Bordé and C. Lämmerzahl, Ann. Phys. (Leipzig), 8, 83 (1999)

[13] S. Gupta, K. Dieckmann, Z. Hadzibabic and D. E. Pritchard, Phys. Rev. Lett. 89, 140401 (2002)
[14] C. Cohen-Tannoudji, J. Dupont-Roc, G. Grynberg, Atom-photon interactions, Wiley Science Paperback (1998)

[15] C. Keller et al., Appl. Phys. B 69, 303 (1999)

[16] C. Champenois et al., Eur. Phys. J. D 13, 271 (2001)

[17] M. Horne, I. Jex and A. Zeilinger, Phys. Rev A 59, 2190 (1999)

[18] M. V. Berry, The diffraction of light by ultrasound, Academic Press, London (1966)

[19] D. Pritchard, private communication (December 2002)

[20] D.M. Giltner, R. W. McGowan and Siu Au Lee, Phys. Rev., A 52, 3966 (1995)

[21] D.M. Giltner, R. W. McGowan and Siu Au Lee, Phys. Rev. Lett., 75, 2638 (1995)

[22] A. E. A. Koolen et al., Phys. Rev. A 65, 041601(R) (2002)

[23] V. S. Letokhov and V. G. Minogin, Zh. Eksp. Teor. Fiz., 74, 1318 (1978)

[24] V. S. Letokhov and V. G. Minogin, Physics Reports, 73, 1 (1981)

[25] Y. Castin and J. Dalibard, Europhys. Lett., 14, 761 (1991)

[26] S. L. Rolston and W. D. Phillips, Nature 416, 219 (2002) 